\title{
Optimal Control of Atlantic Population Canada Geese
}

\author{
${ }^{1}$ Hauser, C.E. ${ }^{2}$ M.C. Runge and ${ }^{3}$ E.G. Cooch \\ ${ }^{1}$ University of Queensland, ${ }^{2}$ Patuxent Wildlife Research Center (Laurel, MD, USA), ${ }^{3}$ Cornell \\ University (Ithaca, NY, USA), E-mail: c.hauser@uq.edu.au
}

Keywords: stochastic dynamic programming; harvest; control; Canada Geese; Branta canadensis; uncertainty; age structure.

\section{EXTENDED ABSTRACT}

The hunting of geese and other waterfowl is an activity that circulates millions of dollars each year in North America. The Atlantic population of Canada Geese (Branta Canadensis) has historically been a major target for hunters throughout the eastern parts of the United States and Canada, although numbers declined significantly in the 1990s. Resident (nonmigratory) populations of geese and migratory populations during the non-breeding season can damage crops and cause public nuisance complaints. Thus, management of goose populations can be a balance between providing high harvest opportunity while not allowing populations to get so large as to cause damage.

We investigate optimal control of the Atlantic population of Canada Geese. We seek to maximize harvest, while maintaining the population within acceptable upper and lower bounds. Control is obtained by setting the harvest rate on breeding adult birds each year. The optimal harvest strategy is informed by the population state, and must incorporate a range of uncertainties regarding population dynamics and the ability to control the population.

The first uncertainty is environmental variation. This is unpredictable and uncontrollable, and represented by stochasticity in breeding productivity from year to year. The second uncertainty considered in this paper is a limited ability to control the population. Given a finite number of hunters, there may be an upper limit on the total number of birds that can be harvested each year. We explore a range of limits to total annual harvest. Structural uncertainty is the third uncertainty considered. Models constructed to represent population dynamics are not a perfect description of the true dynamics. In particular, there is disagreement about the strength of density dependence underlying Canada Goose dynamics. We pose two reasonable but contrasting models of density dependence in this study.

Both models of population dynamics include age structure. Canada Geese, like other goose populations, exhibit life-history attributes that differ by age. Thus, a structured model may be necessary to fully capture the dynamics of this population. Annual harvest decisions are made using the estimated number of birds in each age group.

While age-structured harvest has been investigated in the past, the objective has usually been only to maximize yield. In this study we have the additional goal of maintaining population size within set bounds. Stochastic dynamic programming has rarely been used to optimize the harvest of structured populations, probably due to the increased dimension of the state space required to describe population structure.

Simulations of the optimal harvest under each model show a range of strategies. Under the density independent model, annual harvest is maximized by holding the population size as close as possible to the upper acceptable limit, while ensuring that stochastic fluctuations rarely exceed this limit. When there is limited control, however, the population is optimally maintained at a lower level, to ensure that it does not grow beyond harvest capacity and continue indefinitely with an unacceptably large abundance.

Under the density dependent model, the maximum sustainable yield may be obtained by keeping population size at some level between the minimum and maximum acceptable thresholds. Limits to control do not significantly change this optimal population size, although the amplitude of fluctuations may increase under very limited control. This result depends critically on the fact that the population size that achieves maximum sustainable harvest falls within the desired bounds. If this were not the case, the limits to control could play a more central role.

It is clear that the strength of density dependence and constraints on harvest significantly affect the optimal harvest strategy for this population. Model discrimination might be achieved in the long term, while continuing to meet management goals, by adopting an adaptive management strategy. 


\section{INTRODUCTION}

The sustainable use of wild populations, such as fish and forests, poses significant challenges (Ludwig et al. 1993, Rosenberg et al. 1993). In addition to social, political and economic issues, there are a number of scientific uncertainties that inhibit our ability to identify sustainable exploitation strategies. Populations are influenced by environmental variation, which is unpredictable and uncontrollable. While managers may estimate population size and other relevant information, such as environmental variables, it is impossible to measure the state of the system without error. The harvest rates and other regulations set by managers will not be executed exactly as intended. Finally, the understanding of the population that is used to set regulations (the model) is not a perfect representation of actual population dynamics (Williams et al. 1996).

In representing population dynamics, one of the critical questions is how much structural detail to include. There must be sufficient detail to capture key dynamics, but not so much that estimation issues undermine the predictive ability of the model. One common structural detail that can play a significant role in sustainable harvest is the age composition of the population. Individuals of different ages may contribute differently to the population growth rate through reproduction (motivating the study of reproductive value, MacArthur 1960), thus the age structure of the population can affect growth rate and harvest potential. The consideration of age structure, however, brings added uncertainties. First, the modeling process becomes more complicated by including the size of each age group within the population. This increases the number of population processes that must be described, and the number of parameters to be estimated. Second, it is common for harvest regulations to be set using an estimate of total population size, but it is usually much more difficult to measure the proportion of the population belonging to each age group. Third, the optimal harvest will have age structure, but it is unlikely that the individuals to be harvested can be selected according to age.

In North America, Canada Geese are an important harvested species that exhibit considerable population structure. The Atlantic population, to be modeled in this paper, has a range covering eastern parts of the United States and Canada. Like many species of waterfowl, Canada Geese are hunted for sport, an activity circulating millions of dollars each year. Resident (non-migratory) populations of geese and migratory populations during the non-breeding season can damage crops and cause public nuisance complaints. Thus, management of goose populations can be a balance between providing high harvest opportunity while not allowing populations to get so large as to cause damage.

Canada Geese in the Atlantic population, like other goose populations, exhibit life-history attributes that differ by age. The age at first reproduction is typically greater than three years. There is reason to believe that birds of different ages and breeding status may have different survival rates, including a different vulnerability to harvest. Thus, a structured model may be necessary to fully capture the dynamics of this population, and hence, to derive an appropriate harvest policy.

Before hunting regulations for Atlantic population Canada Geese are set, an aerial survey of breeding grounds is conducted annually in June to estimate the number of breeding pairs of birds. In addition, annual reproduction is estimated from the age-ratio of birds banded in the late summer. The abundance of non-breeding birds in several age classes can be reconstructed from these two data series. Thus, observation of the system state can support decision-making that depends on the age structure of the population.

We use stochastic dynamic programming to find the optimal harvest strategy for this population of Canada Geese, based on the estimated dynamics and the management objectives. This technique has been used for optimizing harvest in the past (Walters 1975, Williams 2001). However, demographic structure (age, size and/or sex) has rarely been considered, probably due to the increased dimension of the state space (an exception is Milner-Gulland 1997).

In this paper, we explore the impact of two key uncertainties on the optimal harvest strategy. First, there is a limited ability to control the population. Given a finite number of hunters, there is an upper limit on the total harvest that can be taken each year. Part of the management objective is to maintain population size below a certain upper bound, so limited control can lead to a risk-averse strategy that foregoes harvest opportunity to insure the population remains within the desired bounds.

Second, there is some disagreement about the strength of density dependence. Over the range of abundance observed for this species, there is no clear information about the carrying capacity of the population. General models of harvested populations indicate that the optimal harvest strategy is sensitive to the strength of density dependence (Reed 1979, Lande et al. 1995), and so we pose two models of reproduction to investigate the potential effects of density dependence. 


\section{MODEL AND METHODS}

\subsection{Management objective}

Most of the literature on optimizing harvest has focused on maximizing the harvest yield. Increasingly, there is concern about the overabundance of some species. Recent examples include raccoons (Rosatte 2000), gulls (Brooks and Lebreton 2001), deer (Doerr et al. 2001, Giles and Findlay 2004, Nugent and Choquenot 2004), moose (Nilsen et al. 2005), and a variety of species of geese (Ankney 1996). While the Atlantic population of Canada Geese is not currently considered to be overabundant, experience with other goose populations raises some concern about the future status of this population. For this reason we set an upper limit on acceptable population size. In addition to this objective, there is a desire to maintain population size above a minimum level needed to sustain a satisfactory level of hunting, and to maximize the opportunity for harvest.

To represent these objectives, we seek to maximize

$$
\sum_{t=0}^{\infty} u\left(N_{t}^{(B)}\right) H_{t}
$$

where

$$
u(N)=\left\{\begin{array}{lc}
1, & N^{\mathrm{MIN}}<N<N^{\mathrm{MAX}} \\
0, & \text { otherwise. }
\end{array}\right.
$$

We use $N_{t}^{(\mathrm{B})}$ to denote the number of breeding adult birds at time $t$, and $H_{t}$ to denote the total harvest taken at time $t$. We set $N^{\text {MIN }}$ and $N^{\text {MAX }}$ as the minimum population size that allows satisfactory harvest and the maximum tolerable population size, respectively. Thus the objective aims to maximize the harvest taken over an infinite time horizon, while keeping the number of breeding adults between $N^{\mathrm{MIN}}$ and $N^{\mathrm{MAX}}$. In practice we will approximate the infinite time horizon problem with a finite time horizon, sufficiently long to ensure that the optimal policy has converged.

\subsection{Decision and state variables}

There is evidence to suggest that there are four significantly different demographic groups within the Canada goose population. These are the 1year-olds (1), 2-year-olds (2), non-breeding adults, (NB) and breeding adults (B). The state of the population is assessed just before the breeding season, so that the youngest individuals are nearing their first birthday and are classified as 1year-olds. Adult birds are three or more years of age, and individuals may or may not breed in a given year.

The control available to management is to set a harvest rate on the breeding adult population. Other groups within the population will be harvested subject to their vulnerability to harvest, relative to breeding adult birds. Young birds are considered to be inexperienced and therefore more easily hunted, along with any accompanying adult parents. Thus the age classes respond to the set harvest rate in different ways. The harvest rate is limited by the total number of birds that can possibly be taken in one year. We set $H^{\mathrm{MAX}}$ to be the maximum total harvest that can be taken in one year.

\subsection{State dynamics}

The number of offspring hatched in spring fluctuates from year to year. There is evidence that it is a function of the timing of the snow melt on breeding grounds. There is disagreement as to whether there is density dependence. We will pose two possible models for breeding productivity $R_{t}$ in year $t$.

In the first model, we assume $R_{t}$ is a random variable that is independent of total population size.

In the second model, we assume that the impact of the timing of snow melt, $z_{t}$, can be described as a standard Normal random variable. Breeding productivity has the density dependent function

$$
R_{t}=\frac{\exp \left(a+b z_{t}+\varepsilon_{t}\right)}{1+\exp \left[c\left(N_{t}^{\mathrm{TOT}}-d\right)\right]},
$$

where $\varepsilon_{t}$ is a Normal process error term, and $N_{t}^{\text {TOT }}$ is the total population size at time $t$. We assume that $a, b, c, d>0$ are known constants and that $\varepsilon_{t}$ has a mean of zero and known variance $\sigma_{\varepsilon}^{2}$. Conditional on knowing total population size, $R_{t}$ has a lognormal distribution, with parameters

$$
\begin{aligned}
& \mu=a-\ln \left(1+\exp \left[c\left(N_{t}^{\mathrm{TOT}}-d\right)\right]\right), \\
& \sigma^{2}=b^{2}+\sigma_{\varepsilon}^{2} .
\end{aligned}
$$

After using one of the above models to calculate breeding productivity, we calculate the number of offspring in year $t$ as

$$
N_{t}^{(0)} \quad=R_{t} N_{t}^{(\mathrm{B})} .
$$


We assume that the harvest rate on breeding adults in year $t$ is set to be $h_{t}^{\left({ }^{(B)}\right.}$. Let the relative vulnerability of offspring and non-breeding birds be $d^{(0)}$ and $d^{(\mathrm{NB})}$, respectively. Then the harvest rates on offspring and non-breeding birds are

$$
\begin{aligned}
& h_{t}^{(0)}=\min \left\{d^{(0)} h_{t}^{(\mathrm{B})}, 1\right\}, \\
& h_{t}^{(\mathrm{NB})}=\min \left\{d^{(\mathrm{NB})} h_{t}^{(\mathrm{B})}, 1\right\} .
\end{aligned}
$$

The total harvest prescribed for each group then becomes

$$
\begin{array}{ll}
H_{t}^{(0)} & =h_{t}^{(0)} N_{t}^{(0)}, \\
H_{t}^{(\mathrm{NB})} & =h_{t}^{(\mathrm{NB})}\left[N_{t}^{(1)}+N_{t}^{(2)}+N_{t}^{(\mathrm{NB})}\right] \\
H_{t}^{(\mathrm{B})} & =h_{t}^{(\mathrm{B})} N_{t}^{(\mathrm{B})} .
\end{array}
$$

The total harvest prescribed for the population is

$$
H_{t} \quad=H_{t}^{(0)}+H_{t}^{(\mathrm{NB})}+H_{t}^{(\mathrm{B})} .
$$

However, we are limited to removing at most $H^{\mathrm{MAX}}$ birds from the population. If the total harvest prescribed by our harvest rate $h_{t}^{(\mathrm{B})}$ exceeds $H^{\mathrm{MAX}}$, then we set the total harvest to be $H^{\mathrm{MAX}}$. We must also rescale our actual harvest rates by multiplying equations (3) to (5) by $H^{\mathrm{MAX}} / H_{t}$.

The survival rates of birds in each demographic group are calculated by combining natural mortality with the harvest rates:

$$
\begin{array}{ll}
s_{t}^{(0)} & =s^{(0)}\left(1-h_{t}^{(0)}\right) \\
S_{t}^{(1)} & =s^{(1)}\left(1-h_{t}^{(\mathrm{NB})}\right) \\
S_{t}^{(2)} & =s^{(2)}\left(1-h_{t}^{(\mathrm{NB})}\right) \\
S_{t}^{(\mathrm{NB})} & =s^{(\mathrm{NB})}\left(1-h_{t}^{(\mathrm{NB})}\right) \\
S_{t}^{(\mathrm{B})} & =s^{(\mathrm{B})}\left(1-h_{t}^{(\mathrm{B})}\right),
\end{array}
$$

where $s^{(i)}$ is the proportion of birds in demographic group $i$ that survive natural mortality, and $S_{t}^{(i)}$ is the proportion of birds in demographic group $i$ that survive both natural mortality and harvest during year $t$.

Then the population at the beginning of year $t+1$ is composed of

$$
N_{t+1}^{(1)}=S_{t}^{(0)} N_{t}^{(0)}
$$

(8) $N_{t+1}^{(\mathrm{NB})}=(1-P)\left(S_{t}^{(2)} N_{t}^{(2)}+S_{t}^{(\mathrm{NB})} N_{t}^{(\mathrm{NB})}+S_{t}^{(\mathrm{B})} N_{t}^{(\mathrm{B})}\right)$

$$
\text { (9) } N_{t+1}{ }^{(\mathrm{B})}=P\left(S_{t}^{(2)} N_{t}^{(2)}+S_{t}^{(\mathrm{NB})} N_{t}^{(\mathrm{NB})}+S_{t}^{(\mathrm{B})} N_{t}^{(\mathrm{B})}\right) \text {, }
$$

where $P$ is the proportion of adults that breed in a given year.

\subsection{Optimization}

We used stochastic dynamic programming to find the optimal state dependent harvest policy. This was carried out in the ASDP software package (Lubow 1995).

We chose a sufficiently long time horizon $T$ to ensure that our state-dependent policy had converged to the stationary policy. There is no payoff in the final time period, so that

$$
V\left(N^{(1)}, N^{(2)}, N^{(\mathrm{NB})}, N^{(\mathrm{B})}, T\right)=0,
$$

for all $N^{(1)}, N^{(2)}, N^{(\mathrm{NB})}, N^{(\mathrm{B})} \geq 0$. We use $V\left(N^{(1)}\right.$, $\left.N^{(2)}, N^{(\mathrm{NB})}, N^{(\mathrm{B})}, t\right)$ to denote the value of choosing the optimal harvest policy from time $t$ to the time horizon, given that the population is composed of $N^{(1)} 1$-year-old birds, $N^{(2)} 2$-year-old birds, $N^{(\mathrm{NB})}$ non-breeding adult birds and $N^{(\mathrm{B})}$ breeding adult birds in year $t$. The optimal state-dependent policy in year $t$ is calculated as the harvest rate $h_{t}^{(\mathrm{B})}$ that maximizes the sum of returns accrued during year $t$ (with respect to our objective function) and expected future returns (the value function $V$, weighted by the probability of each future state, as determined by the state dynamics).

The method of stochastic dynamic programming requires us to discretize the state, decision and random variables. The values used in this paper are listed in Table 1 . To prevent inappropriate extrapolation, a ceiling was placed on population size in equations (6) to (9) in the case of density independent breeding productivity. Other parameter values required for the model are listed in Table 2.

\section{RESULTS}

\subsection{Density-independent model}

In the absence of harvest, the density-independent model for breeding productivity causes the number of breeding adults in the population to grow without bound. When we impose a ceiling on each age group (Table 1), the number of breeding adults maintains this ceiling over time. Other age groups fluctuate below their ceiling, although the number of 1-year-old birds reaches its ceiling on occasions. The total population size fluctuates between 2 and 2.6 million birds.

The optimal harvest strategy was derived for a variety of values of $H^{\mathrm{MAX}}$ (Table 1). Each harvest 
Table 1. Values taken by state, decision and random variables for the optimization. Notation $X: Y: Z$ indicates that the minimum value considered is $X$, increasing in steps of size $Y$ to a maximum value $Z$.

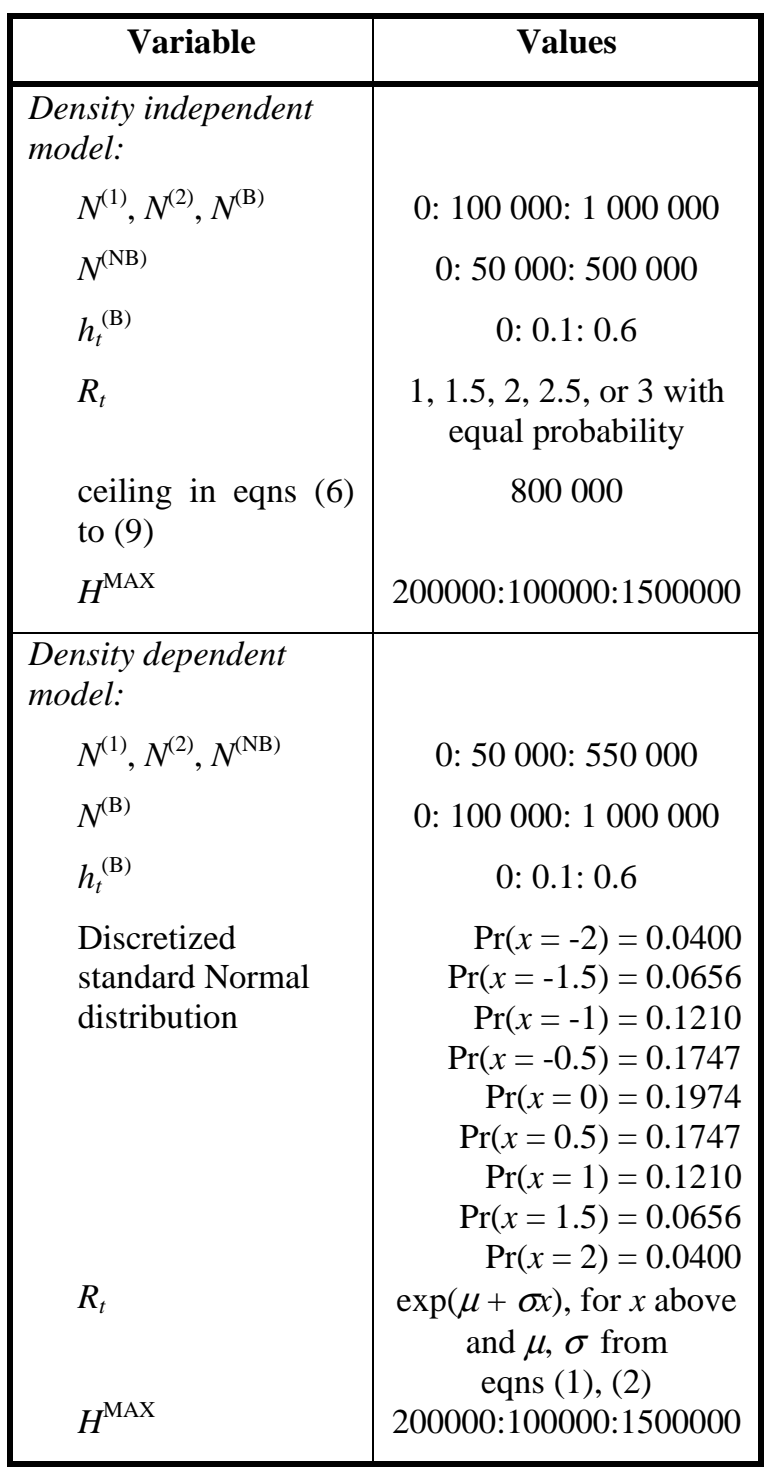

Table 2.Values for parameters in the optimization.

\begin{tabular}{|l|r|l|r|}
\hline Parameter & \multicolumn{1}{|c|}{ Value } & Parameter & Value \\
\hline$N^{\mathrm{MIN}}$ & 120000 & $d^{(0)}$ & 2.0 \\
$N^{\mathrm{MAX}}$ & 500000 & $d^{(\mathrm{NB})}$ & 1.0 \\
$a$ & 0.7 & $s^{(0)}$ & 0.65 \\
$b$ & 0.15 & $s^{(1)}$ & 0.86 \\
$c$ & $3.0 \times 10^{-6}$ & $s^{(2)}$ & 0.86 \\
$d$ & 800000 & $s^{(\mathrm{NB})}$ & 0.86 \\
$\sigma_{\varepsilon}$ & 0.2 & $s^{(\mathrm{B})}$ & 0.86 \\
$P$ & 0.8 & & \\
\hline
\end{tabular}

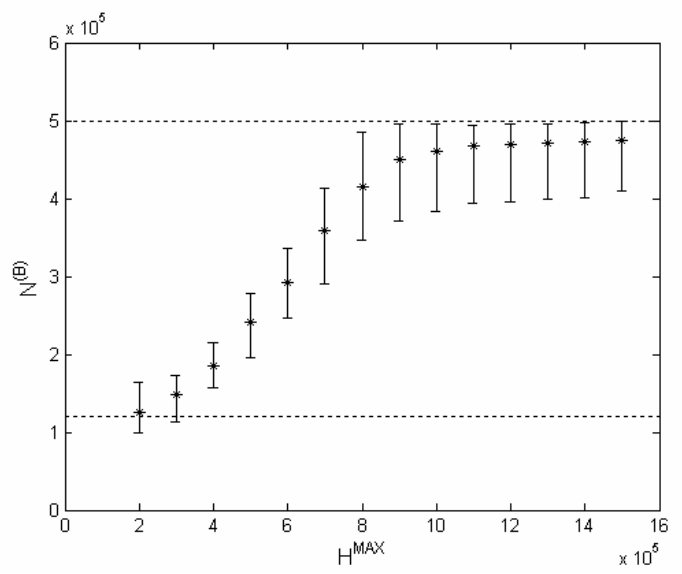

Figure 1. Long-term breeding population size under the optimal harvest strategy, as a function of maximum annual harvest $H^{\mathrm{MAX}}$, using the density independent model for breeding productivity.

Asterisks denote the mean population size over 100 simulations; error bars indicate the interval that covers $95 \%$ of observations. Dotted lines show the lower and upper acceptable thresholds $N^{\mathrm{MIN}}$ and $N^{\mathrm{MAX}}$, respectively.

strategy was simulated for 100 runs, each 200 years in length. The first 100 years in each run were discarded. In Figure 1 we display the mean number of breeding adults in the population and the fluctuation around the mean for the remaining observations.

When $H^{\mathrm{MAX}}$ was set to 200000 , the population was not always successfully held within the desired range, i.e. a breeding population size between $N^{\mathrm{MIN}}$ and $N^{\mathrm{MAX}}$. In 90 of the 100 runs, the population grew until the number of breeding adults reached its ceiling (Table 1). These runs were discarded from the data plotted in Figure 1.

Since population growth is independent of density in the range of interest, harvest (and hence the objective) is maximized when the population is large. We see in Figure 1 that when there is a great capacity to harvest the population $\left(H^{\mathrm{MAX}}\right.$ is large), then the population is maintained at a level close to the upper acceptable threshold $N^{\mathrm{MAX}}$. Stochasticity in breeding productivity $R$ means that population size will fluctuate, and the mean breeding population size is held a little below $N^{\mathrm{MAX}}$ to reduce the risk of fluctuations above $N^{\mathrm{MAX}}$. However when ability to control is limited ( $H^{\mathrm{MAX}}$ is low), then the breeding population size is held well below the maximum acceptable level. If the population were permitted to grow nearer to $N^{\mathrm{MAX}}$, the maximum harvest $H^{\mathrm{MAX}}$ could not prevent the population from continuing to grow beyond $N^{\mathrm{MAX}}$, so that the objective is not achieved in the long term. 
(a)

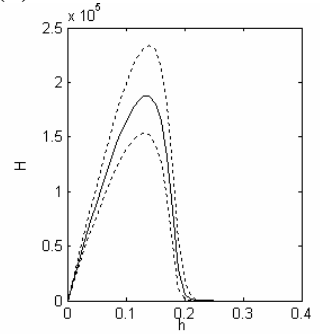

(b)

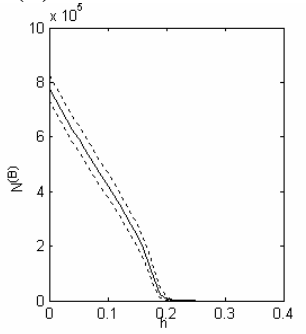

Figure 2. Simulation of the population with density dependent breeding productivity under a constant harvest rate: (a) annual harvest, and (b) breeding population size, as a function of harvest rate $h^{(\mathrm{B})}$. Mean values are given by solid lines, $95 \%$ of observations fall between the dotted lines.

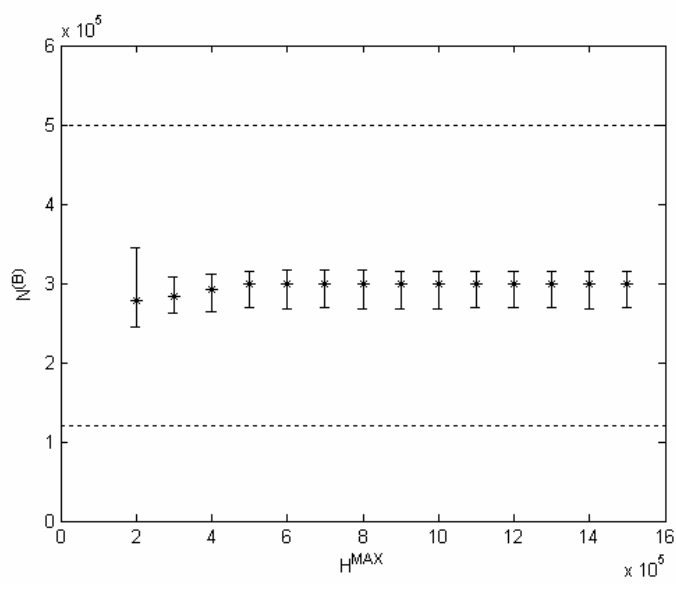

Figure 3. Long-term breeding population size under the optimal harvest strategy, as a function of maximum annual harvest $H^{\mathrm{MAX}}$, using the density

dependent model for breeding productivity.

Asterisks denote the mean population size over 100 simulations; error bars indicate the interval that covers $95 \%$ of observations. Dotted lines show the lower and upper acceptable thresholds $N^{\mathrm{MIN}}$ and $N^{\mathrm{MAX}}$, respectively.

\subsection{Density-dependent model}

In the absence of harvest, the population under density dependent breeding productivity approaches a stochastic carrying capacity above the maximum acceptable population size $N^{\mathrm{MAX}}$. Using the parameters given in Table 2, the breeding population fluctuates around 700 to 800 thousand birds, while the total population size is 1.2 to 1.4 million birds.

In Figure 2 we present the maximum sustainable yield for this population, assuming total harvest control ( $H^{\mathrm{MAX}}$ is infinite). These plots were created by simulating the population under a fixed harvest rate $h^{(\mathrm{B})}$, making 100 runs, each 200 years in length, and discarding the first 100 observations. The annual yield and breeding population size were recorded. Figure 2(a) indicates that annual yield is maximized for a harvest rate of about $13 \%$, which holds the long term breeding population size at a little over 300000 birds (Figure 2 (b)).

State-dependent optimization and simulations were run in the same manner as for the density independent model. (Variable levels and parameter values are listed in Tables 1 and 2, respectively.) The number of breeding adults did not exceed $N^{\mathrm{MAX}}$ in any simulation. Figure 3 displays the mean breeding population size and fluctuation around the mean, under a variety of levels of $H^{\mathrm{MAX}}$ (Table 1). For all levels of control above $H^{\mathrm{MAX}}=500000$, the breeding population is held at about 300000 birds with some fluctuation due to stochasticity in breeding productivity. When $H^{\mathrm{MAX}}=300000$ or 400000 the population is held slightly lower to ensure that control is maintained. When $H^{\mathrm{MAX}}=200000$, population fluctuations are much larger. With such limited control it is difficult to maintain the population at the level giving maximum sustainable yield. However the density dependent function for breeding productivity ensures that the population never exceeds its upper acceptable threshold $N^{\mathrm{MAX}}$. This result depends critically on the fact that the population size that achieves maximum sustainable harvest falls within the desired bounds. If the nature of density-dependence in the population did not have this property, then there would be tension between the different components of the objective, and the limits to control could play a more central role.

\section{CONCLUSIONS}

In this paper we have explored a variety of models for the optimal control of Canada geese, all of which may reasonably describe the dynamics of the Atlantic population and the constraints on harvest. We posed two models for stochastic breeding productivity, and investigated a variety of values for the upper limit on annual harvest. The objective was to maximize harvest, while maintaining the breeding population size between specified limits $N^{\mathrm{MIN}}$ and $N^{\mathrm{MAX}}$.

The optimal harvest strategy looks markedly different over the range of alternative models chosen. Under the density independent model, annual harvest increases linearly with breeding population size. When there is sufficient control available ( $H^{\mathrm{MAX}}$ is sufficiently large) then it is optimal to maintain the breeding population as close to $N^{\mathrm{MAX}}$ as possible, while ensuring that 
fluctuations rarely exceed this value. When control is limited then the population is optimally maintained at a lower level, to ensure that it does not grow beyond harvest capacity and $N^{\mathrm{MAX}}$ indefinitely.

Under the density dependent model for breeding productivity, the maximum sustainable yield is obtained by keeping the breeding population size well below the maximum acceptable level $N^{\mathrm{MAX}}$. This level appears optimal for almost all levels of maximum control $H^{\mathrm{MAX}}$. However the amplitude of fluctuations may increase under very limited control.

Achievement of management goals could clearly be improved with a better understanding of population dynamics and limits to annual harvest. The data collection required to discriminate between these alternative models is a very slow process, but might be sped up by experimental management. The use of adaptive management techniques (Walters 1986, Williams et al. 1996) can find the optimal trade-off between meeting management goals in the short term and experimenting for learning in the long term.

\section{ACKNOWLEDGMENTS}

This study was funded by the U.S. Geological Survey State Partnership Program, the Atlantic Flyway Council, Cornell University, and the New York State Department of Environmental Conservation. CEH's $\mathrm{PhD}$ scholarship is provided by the Australian Research Council.

\section{REFERENCES}

Ankney, C.D. (1996), An embarrassment of riches: too many geese, Journal of Wildlife Management, $60,217-223$.

Brooks, E.N. and J.-D. Lebreton (2001), Optimizing removals to control a metapopulation: application to the yellow legged herring gull (Larus cachinnans), Ecological Modelling, 136, $269-284$

Doerr M.L., J.B. McAninch, and E.P. Wiggers (2001), Comparison of 4 methods to reduce whitetailed deer abundance in an urban community, Wildlife Society Bulletin, 29, 1105 - 1113.

Giles, B.G. and C.S. Findlay (2004), Effectiveness of a selective harvest system in regulating deer populations in Ontario, Journal of Wildlife Management, 68, 266 - 277.

Lande, R., S. Engen, and B.-E. Saether (1995), Optimal harvesting of fluctuating populations with a risk of extinction, The American Naturalist, 145, $728-745$.
Lubow, B. (1995), SDP: Generalized software for solving stochastic dynamic optimization problems, Wildlife Society Bulletin, 23, 738 - 742.

Ludwig, D., R. Hilborn, and C. Walters (1993), Uncertainty, resource exploitation, and conservation: lessons from history, Science, 260, 17, 36.

McArthur, R.H. (1960), On the relation between reproductive value and optimal predation, Proceedings of the National Academy of Sciences of the United States of America, 46, 143 - 145.

Milner-Gulland, E.J. (1997), A stochastic dynamic programming model for the management of the saiga antelope, Ecological Applications, 7, 130 142.

Nilsen E.B., T. Pettersen, H. Gundersen, J.M. Milner, A. Mysterud, E.J. Solberg, H.P. Andreassen, and N.C. Stenseth (2005), Moose harvesting strategies in the presence of wolves, Journal of Applied Ecology, 42, 389 - 399.

Nugent, G. and D. Choquenot (2004), Comparing cost-effectiveness of commercial harvesting, statefunded culling, and recreational deer hunting in New Zealand, Wildlife Society Bulletin, 32, 481 492.

Reed, W.J. (1979), Optimal escapement levels in stochastic and deterministic harvesting models, Journal of Environmental Economics and Management, 6, 350 - 363.

Rosatte, R.C. (2000), Management of raccoons (Procyon lotor) in Ontario, Canada: Do human intervention and disease have significant impact on raccoon populations? Mammalia, 64, 369 - 390.

Rosenberg, A.A., M.J. Fogarty, M.P. Sissenwine, J.R. Beddington, and J.G. Shepherd (1993), Achieving sustainable use of renewable resources, Science, 262, 828 - 829.

Walters, C.J. (1975), Optimal harvest strategies for salmon in relation to environmental variability and uncertain production parameters, Journal of the Fisheries Research Board of Canada, 32, 1777 1784.

Walters, C. J. (1986), Adaptive Management of Renewable Resources, Blackburn Press, 374pp., Caldwell, NJ.

Williams, B.K., F.A. Johnson, and K. Wilkins (1996), Uncertainty and the adaptive management of waterfowl harvests, Journal of Wildlife Management, 60, 223 - 232.

Williams, B.K. (2001), Uncertainty, learning, and the optimal management of wildlife, Environmental and Ecological Statistics, 8, 269 288. 